\title{
Effects of the Montague resonance on the formation of the beam distribution during multiturn injection painting in a high-intensity proton ring
}

\author{
H. Hotchi ${ }^{*}$ \\ Accelerator division, J-PARC center, Japan Atomic Energy Agency (JAEA), \\ Tokai, Naka, Ibaraki, 319-1195 Japan
}

(Received 24 June 2019; revised manuscript received 2 April 2020; accepted 23 April 2020; published 8 May 2020)

\begin{abstract}
The "Montague resonance" is a familiar issue in high-intensity proton rings, which causes emittance exchange between the two transverse planes, having a significant influence on their high-intensity performance. In this paper, we discuss the characteristic effects of this resonance on the formation of the beam distribution during multiturn injection painting that are measured experimentally at the $3-\mathrm{GeV}$ rapid cycling synchrotron in the Japan Proton Accelerator Research Complex. In addition, we discuss an optimization methodology for injection painting for the case involving emittance exchange.
\end{abstract}

DOI: 10.1103/PhysRevAccelBeams.23.050401

\section{INTRODUCTION}

In high-intensity proton accelerator facilities, controlling space charge induced emittance blow-up and beam loss is always a critical challenge in maximizing their high-intensity performance while maintaining the hands-on-maintenance environment. Among various space charge induced phenomena, the "Montague resonance," which is the main subject of this paper, is one of the most familiar space-charge issues in high-intensity proton rings. The resonance is well known to cause emittance exchange between the horizontal and vertical planes when the splitting between the horizontal and vertical betatron tunes is small $\left(\nu_{x} \cong \nu_{y}\right)$, possibly imposing a limit on the achievable beam intensity.

Through a detailed single-particle analysis, B.W. Montague first pointed out that the space-charge-driven fourth-order difference resonance, $2 \nu_{x}-2 \nu_{y}=0$, causes emittance exchange [1]. As shown in the Appendix, if we assume a Gaussian distribution and the condition of small tune splitting, the perturbation Hamiltonian arising from the space charge is approximately given in terms of the conjugate angle $\left(\varphi_{x, y}\right)$ and action $\left(J_{x, y}\right)$ variables as follows:

$$
\begin{aligned}
H_{1}\left(\varphi_{x}, J_{x}, \varphi_{y}, J_{y} ; s\right)= & V_{1100} J_{x}+V_{0011} J_{y} \\
& +V_{2200} J_{x}^{2}+V_{0022} J_{y}^{2}+V_{1111} J_{x} J_{y} \\
& +2 V_{2002} J_{x} J_{y} \cos \left[2\left(\nu_{x}-\nu_{y}\right) \theta\right. \\
& \left.+2\left(\varphi_{x}-\varphi_{y}\right)\right],
\end{aligned}
$$

"hotchi.hideaki@jaea.go.jp

Published by the American Physical Society under the terms of the Creative Commons Attribution 4.0 International license. Further distribution of this work must maintain attribution to the author(s) and the published article's title, journal citation, and DOI. where $s$ is the longitudinal position along the closed ring orbit; $\theta=s / R$ is the orbiting angle, where $R$ is the ring radius; and $V_{1100}, V_{0011}, V_{2200}, V_{0022}, V_{1111}$, and $V_{2002}$ are constant coefficients, which are given by Eqs. (A17)(A22). From Hamilton's equations of motion, we obtain

$$
\frac{d J_{x}}{d s}+\frac{d J_{y}}{d s}=-\frac{\partial H_{1}}{\partial \varphi_{x}}-\frac{\partial H_{1}}{\partial \varphi_{y}}=0
$$

which yields

$$
J_{x}+J_{y}=\text { const }
$$

Here, the quantities $2 \times J_{x, y}$ can be interpreted as singleparticle emittances. That is, the physical significance of Eq. (3) is that the emittance of a single particle is exchanged periodically between the two transverse planes as a result of the fourth-order single-particle difference resonance driven by the nonlinear space-charge coupling force (the $J_{x} J_{y}$ term in the Hamiltonian $H_{1}$ ).

In addition to such a single-particle (incoherent) mechanism, the Montague resonance also occurs as a coherent phenomenon caused by the time-varying space-charge force induced in the presence of internal energy anisotropy [2-4]. It cannot be found in the above single-particle picture which assumes a frozen space-charge potential. In order to explore a coherent phenomenon, self-consistent modeling is required. To this end, self-consistent particlein-cell simulation studies have actively been carried out. A typical example of a coherent phenomenon is the occurrence of rms emittance exchange in a KapchinskyVladimirsky (KV) beam with the initial anisotropy $\epsilon_{x} / \epsilon_{y} \neq$ 1 for the horizontal and vertical $r m s$ emittances $[3,4]$. The KV distribution has a uniform charge density [5] and no driving term for the fourth-order single-particle resonance. 
Therefore, the emittance exchange found for a $\mathrm{KV}$ beam is not predictable in the single-particle picture. It can be explained only in terms of a coherent difference mode resonance driven by the anisotropy between the two transverse planes, which makes the nonlinear space-charge coupling term grow exponentially from the noise level, driving the emittance exchange [2-4].

Thus, the mechanism of the Montague resonance can be considered as a combination of coherent and incoherent resonance phenomena; e.g., an anisotropic water-bag or Gaussian distribution not only has a coherent phenomenon, but also involves an incoherent resonance phenomenon due to a space-charge coupling term existing from the beginning [3]. These effects of the resonance are avoidable by sufficiently splitting the horizontal and vertical tunes. But, the tune space near the Montague resonance has an aspect that it is favorable for avoiding more serious structure resonances. Actually, several facilities set the tunes in the vicinity of the Montague resonance for this reason, although the beam is heavily influenced by emittance exchange. The Montague resonance is a major issue for such high-intensity proton machines, and its influence on the high-intensity performance is being studied actively even now [6,7]. However, most of these studies have been conducted assuming simple initial beam distributions such as $\mathrm{KV}$, Gaussian, or water-bag distributions. Consequently, knowledge of various aspects of the Montague resonance that accompany actual beam operations is limited.

The scope of the present study is to investigate the possible effects of the Montague resonance in the process of "multiturn injection painting"; that is, to reveal new aspects of the Montague resonance in such a "dynamical" situation. Injection painting enables us to form a different spatial particle distribution from multiturn injected beams. It is an essential technique for space-charge mitigation, and optimizing it, that is, establishing a stable low-loss beam distribution by injection painting is a critical issue for highintensity proton rings $[8,9]$.

In this paper, we investigate the influences of the Montague resonance on the formation of the beam distribution during multiturn injection painting, and further discuss an optimization methodology for injection painting for the case involving emittance exchange, using numerical simulation results backed up by experimental results obtained from a 1-MW high-power beam operation (providing $8.33 \times 10^{13}$ protons per pulse at $25 \mathrm{~Hz}$ ) at the $3-\mathrm{GeV}$ Rapid Cycling Synchrotron (RCS [10-12]) in the Japan Proton Accelerator Research Complex (J-PARC [10]).

\section{OUTLINE OF J-PARC 3-GeV RCS AND RESEARCH BACKGROUND}

\section{A. J-PARC 3-GeV RCS}

The J-PARC 3-GeV RCS is a world leading high-power pulsed proton driver, which has the goal of achieving a 1-MW

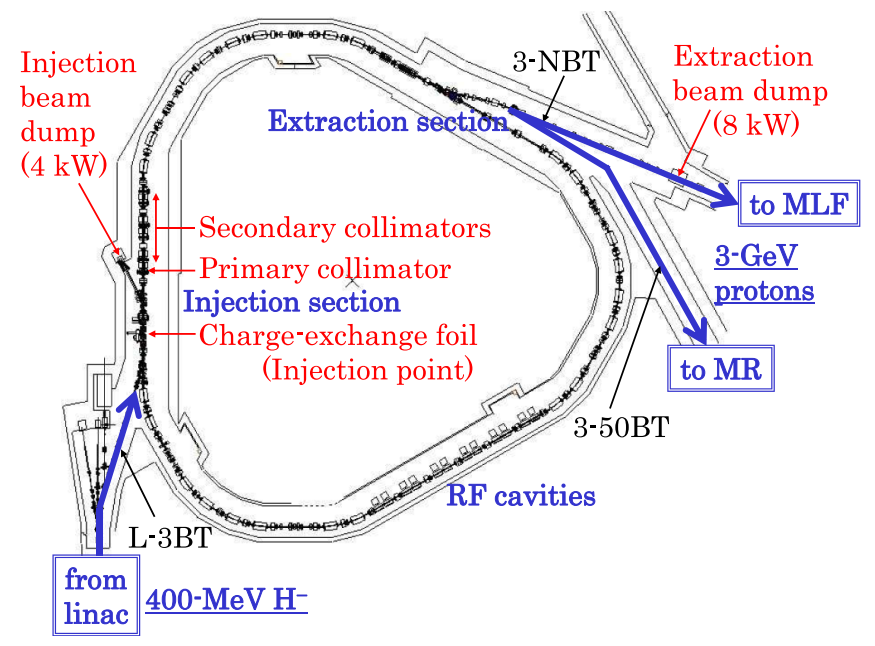

FIG. 1. Schematic view of the J-PARC 3-GeV RCS.

beam power $\left(8.33 \times 10^{13}\right.$ protons per pulse) [10-12]. As shown in Fig. 1, a 400-MeV negative hydrogen ion $\left(\mathrm{H}^{-}\right)$ beam from the injector linac is delivered to the RCS injection point, where it is multiturn charge-exchange injected into the RCS through a carbon foil over a period of $0.5 \mathrm{~ms}$ (corresponding to 307 turns). The RCS accelerates the injected protons up to $3 \mathrm{GeV}$ with a repetition rate of $25 \mathrm{~Hz}$. Most of the RCS beam pulses are delivered to the Materials and Life Science Experimental Facility (MLF) to produce pulsed muons and neutrons, while only four pulses in every several seconds (2.48 or $5.20 \mathrm{~s}$ ) are injected into the following 30GeV Main Ring (MR), switching the beam destination pulse by pulse.

The requirements are different for the beams delivered to the MLF and to the MR. Therefore, different parameter optimizations are required for the two modes of operation [13]. Due to the higher operational duty factor, the machine activation levels of the RCS are mainly determined by the beam operation to the MLF. Consequently, the dominant requirement in this operating mode is to control beam loss. In addition, the MLF requires a large emittance beam with low charge density in order to mitigate the shockwave on the neutron production target, which is essential to prolong its operational lifetime. On the other hand, in contrast to the MLF, the MR requires a small emittance beam with low beam halo, which is essential to mitigate beam loss in the MR. In order to meet the different requirements for the beams delivered to the MLF and to the MR, we have utilized transverse injection painting, employing large painting for the MLF and small painting for the MR.

\section{B. Transverse injection painting}

Figure 2 shows a schematic diagram of the transverse injection painting scheme [8] that is applied at the RCS. As shown in the figure, horizontal painting is performed by a horizontal closed orbit variation during injection. In this way, the injection beam is filled from the middle to the 


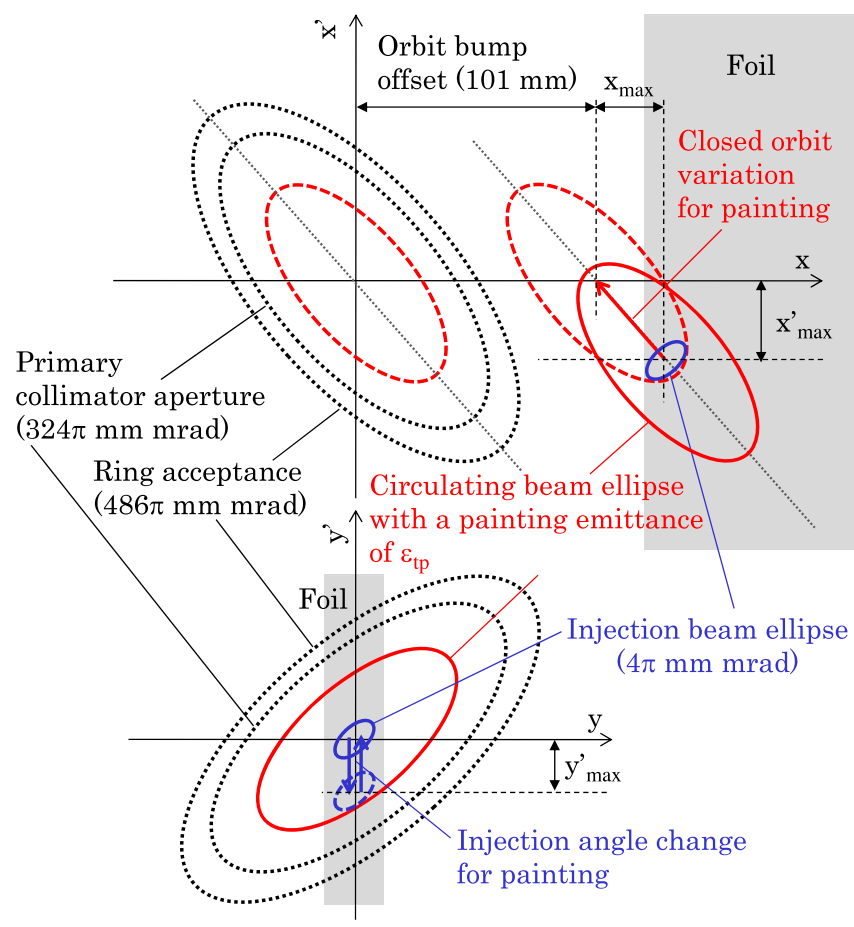

FIG. 2. Schematic illustration of the transverse injection painting scheme that is applied at the RCS. The top panel is for the horizontal phase space, and the bottom panel is for the vertical case.

outside on the horizontal phase space. On the other hand, vertical painting is performed by a vertical injection angle change during injection. The direction of the change in angle is reversible, so the injection beam can be painted either from the middle to the outside, or from the outside to the middle on the vertical phase space.

For this painting process, the phase-space offset of the injection beam relative to the closed ring orbit is varied according to the square-root-type functions:

$$
x_{\text {paint }}=x_{\max } \sqrt{t / T}, x_{\text {paint }}^{\prime}=-x_{\max }^{\prime} \sqrt{t / T},
$$

for the horizontal plane, and

$$
\begin{aligned}
& y_{\text {paint }}=0, \\
& y_{\text {paint }}^{\prime}=-y_{\max }^{\prime} \sqrt{t / T},
\end{aligned}
$$

or

$$
\begin{aligned}
& y_{\text {paint }}=0, \\
& y_{\text {paint }}^{\prime}=-y_{\text {max }}^{\prime} \sqrt{1-t / T},
\end{aligned}
$$

for the vertical plane. Here $\left(x_{\max }, x_{\max }^{\prime}\right)$ and $\left(0, y_{\max }^{\prime}\right)$ are, respectively, the maximum phase-space offsets in the horizontal and vertical planes, for which the injection beam ellipse is inscribed at the edge of the circulating beam ellipse with a required painting emittance. The other parameters are the injection duration $T=0.5 \mathrm{~ms}$ and the time step $t$, which increases from $t=0$ through the end of injection. These square-root-type painting functions theoretically make a uniform charge density distribution in the transverse phase space.

The combination of the horizontal painting given by Eq. (4) and the vertical painting from Eq. (5), in which the injection beam is distributed along the line $J_{x}-J_{y}=0$ in the action space of the beam, is known as "correlated painting." Another combination of Eqs. (4) and (6) is known as "anticorrelated painting," for which the injection beam is painted along the line $J_{x}+J_{y}=$ const. That is, it forms an approximate $\mathrm{KV}$ distribution in theory. As to the painting emittance $\epsilon_{t p}$, it is adjustable up to $200 \pi \mathrm{mm} \mathrm{mrad}$ on the horizontal and vertical planes independently, where $\epsilon_{t p}$ is defined as the un-normalized value of the entire painting area.

\section{Operating point}

Figure 3 shows the tune diagram, in which the blue lines represent the structure resonances up to fourth order, derived from the three-fold symmetric lattice of the RCS. In the present work, the operating point was set in the vicinity of $\left(\nu_{x}=6.45, \nu_{y}=6.42\right)$, as shown by the black circle in the figure.

This operating point allows space-charge tune shifts to avoid serious structure resonances, such as low-order onedimensional resonances and coupling-sum resonances, which are directly connected with beam loss through a critical emittance growth. In addition, the effects of the lattice imperfections, which are listed in the next section, are also well minimized there. But, in exchange for those, this operating point is very close to the Montague

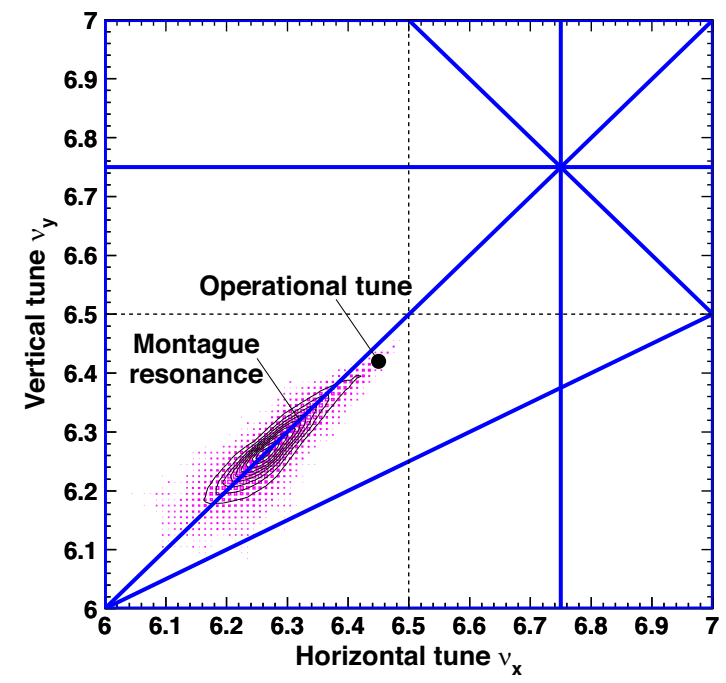

FIG. 3. Tune diagram near the operating point (black circle), in which the blue lines show the structure resonances up to fourth order, derived from the three-fold symmetric lattice of the RCS. 
resonance. Therefore, the beam suffers strongly from space charge induced emittance exchange. In other words, the major source of emittance growth and beam loss there is limited to the Montague resonance arising from space charge; we can investigate the effect of the Montague resonance on the beam isolating it from other effects at the operating point.

The aim of this paper is to optimize injection painting for the case involving emittance exchange. Below we discuss the optimal configuration, correlated or anticorrelated painting, to meet the requirements for the MLF and the MR, taking into account the evolution of the beam distribution with space charge and associated emittance exchange.

\section{OUTLINE OF NUMERICAL SIMULATION}

In this work, numerical simulation was performed with a particle tracking code called "SIMPSONS" [14]. This code enables us to simulate three-dimensional motions of beam particles including the space-charge effect and the realistic injection painting process. The numerical simulation has well reproduced the experimental data such as beam profile and beam loss in the RCS, and played an essential role in understanding emittance growth and beam loss mechanisms and finding their solution in combination with actual beam experiments. [12].

In this numerical simulation, all the lattice imperfections, which have been identified so far, are included; multipole field components of the main ring magnets, magnetic field errors among the same-family magnets, misalignments, static leakage fields from the extraction beam-line magnets, edge focuses of the injection bump magnets, and so on. They were evaluated through magnetic field measurements and/or beam-based measurements. One of characteristic features of this code is to take "time" as an independent variable, not longitudinal position, which is used more widely. Therefore, time-dependent lattice imperfections as well as time-independent ones can be implemented easily in the simulation.

This code computes the space-charge potential based on a particle-in-cell algorithm with cylindrical meshes in the $(r, z, \varphi)$ coordinates. The fractional charge of each macroparticle is assigned to the nearby grid points following the area-weighting method. The charge distribution at the grid points is Fourier-transformed in the azimuthal direction, and then the Poisson equation is solved in the $(r, z)$ space for each azimuthal mode assuming a boundary condition of the circular cross-sectional beam pipe which is a good approximation for the RCS. Finally, the space-charge force is applied to each macroparticle as a three-dimensional impulse kick. The space-charge potential is recalculated at a given time step out of the macroparticles whose distribution evolves in a self-consistent manner as per the progression of time. The time step applied in the present simulation was $2 \mathrm{~ns}$ during injection and $10 \mathrm{~ns}$ for the others, corresponding to 850 and 170 kicks per turn.

The number of macroparticles was typically set to $5 \times$ $10^{5}$ using a transverse grid of $64(r) \times 64(\varphi)$ for a conducting boundary of $r=0.145 \mathrm{~m}$ and a longitudinal grid of $50(z)$. Careful analyses for the convergence of macroparticle motions confirmed that the use of these numerical parameters is tolerable for obtaining meaningful simulation results [8].

\section{EMITTANCE GROWTH DURING INJECTION PAINTING}

Figure 4 shows the time dependences of the normalized $99 \%$ emittances for the first $1.5 \mathrm{~ms}$, calculated with $\epsilon_{t p}=$ $200 \pi \mathrm{mm} \mathrm{mrad}$ and $\epsilon_{t p}=50 \pi \mathrm{mm} \mathrm{mrad}$-for correlated and anticorrelated painting, respectively-where the $99 \%$ emittance is defined as the emittance value containing $99 \%$ of the total charge. For large painting $\left(\epsilon_{t p}=200 \pi \mathrm{mm} \mathrm{mrad}\right)$, correlated painting generates a critical large emittance growth on the vertical plane. Therefore, for large painting, correlated painting gives a larger beam loss than does anticorrelated painting, as is shown later. But, conversely, for small painting ( $\epsilon_{t p}=50 \pi \mathrm{mm}$ mrad), correlated painting yields smaller beam emittances on both the horizontal and vertical planes than does anticorrelated painting. Thus, correlated and anticorrelated painting provide different situations for large painting and small painting.

These phenomena can be understood by considering the effects of emittance exchange caused by the Montague resonance, as discussed below.

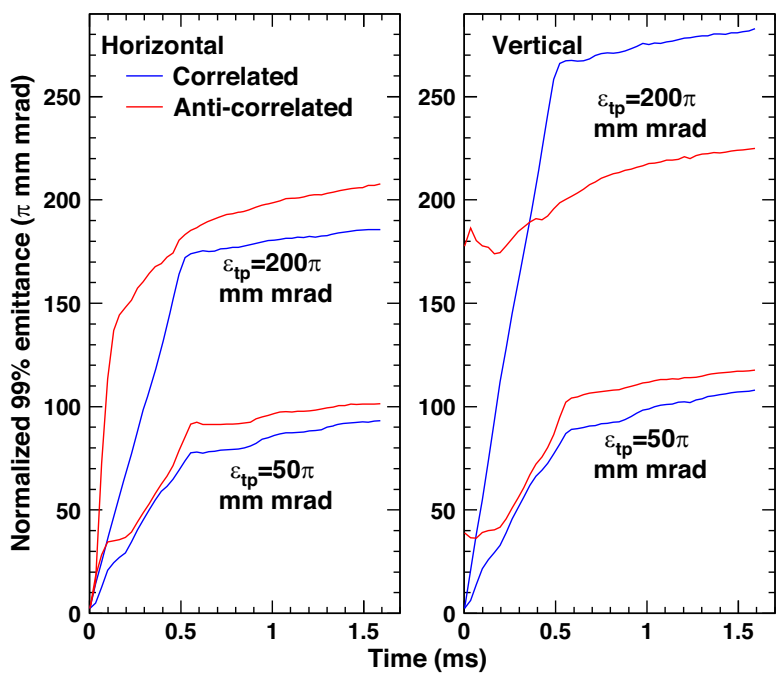

FIG. 4. Numerical simulation results; time dependences of the normalized $99 \%$ emittances for the first $1.5 \mathrm{~ms}$, calculated with $\epsilon_{t p}=200 \pi \mathrm{mm} \mathrm{mrad}$ and $\epsilon_{t p}=50 \pi \mathrm{mm} \mathrm{mrad}$ for correlated and anticorrelated painting, respectively. 


\section{EFFECTS OF EMITTANCE EXCHANGE DURING LARGE PAINTING}

First, we discuss the particle motions during injection painting for a large painting emittance of $\epsilon_{t p}=$ $200 \pi \mathrm{mm}$ mrad, which is required for the beam delivered to the MLF.

Figure 5 shows scatter plots of the single-particle actions $\left(\begin{array}{lll}J_{y} & v s & J_{x}\end{array}\right)$ from the beginning to the end of injection, calculated for correlated painting with $\epsilon_{t p}=200 \pi \mathrm{mm}$ mrad. The left panels correspond to the results obtained with no space charge. As clearly shown there, in correlated painting, the injection beam is painted along the line $J_{x}-J_{y}=0$, i.e., from the middle to the outside on both the horizontal and vertical planes. This situation changes significantly when the space charge is turned on, as shown in the right panels; it can
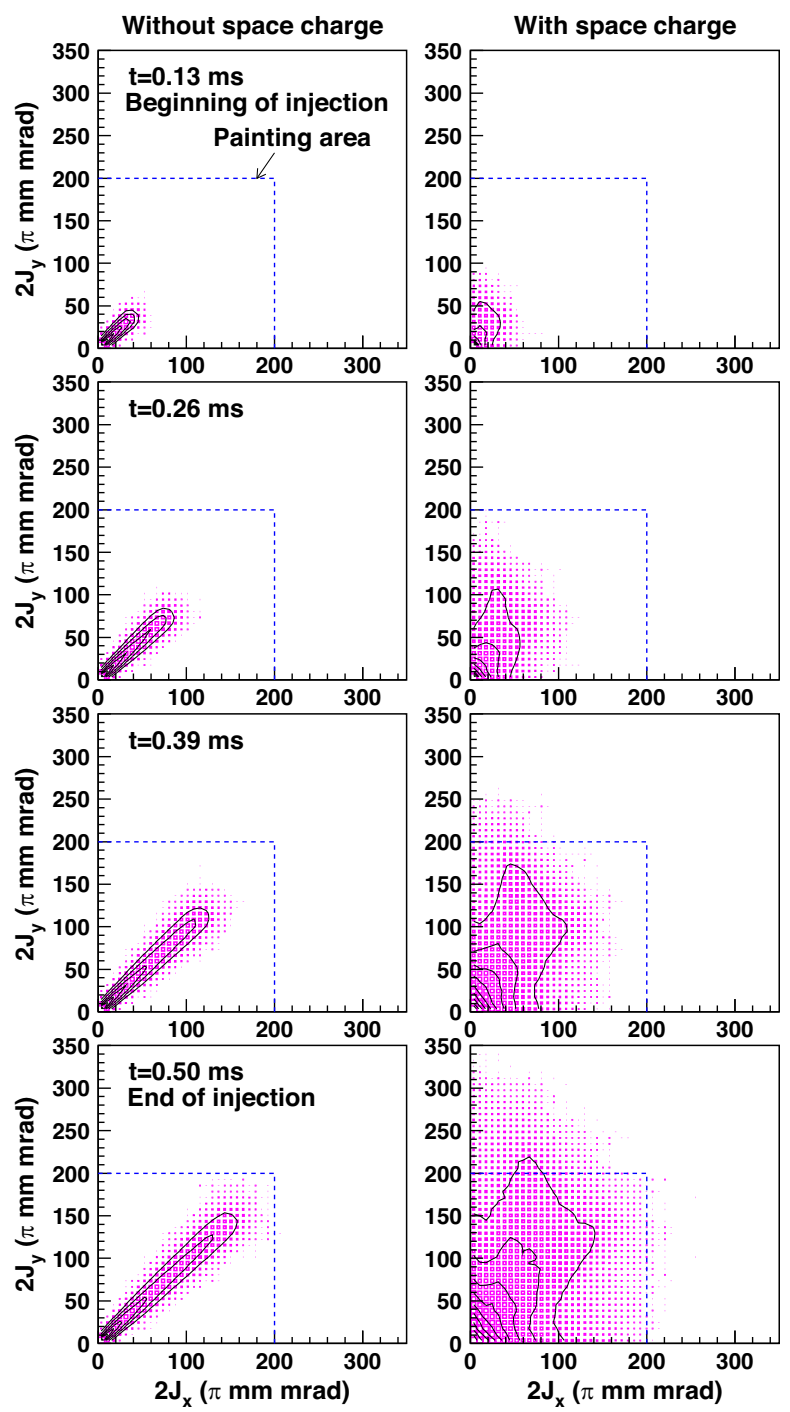

FIG. 5. Numerical simulation results; scatter plots of the singleparticle actions $\left(\begin{array}{llll}J_{y} & v s & J_{x}\end{array}\right)$ from the beginning to the end of injection, calculated for correlated painting with $\epsilon_{t p}=$ $200 \pi \mathrm{mm}$ mrad without (left) and with (right) space charge.

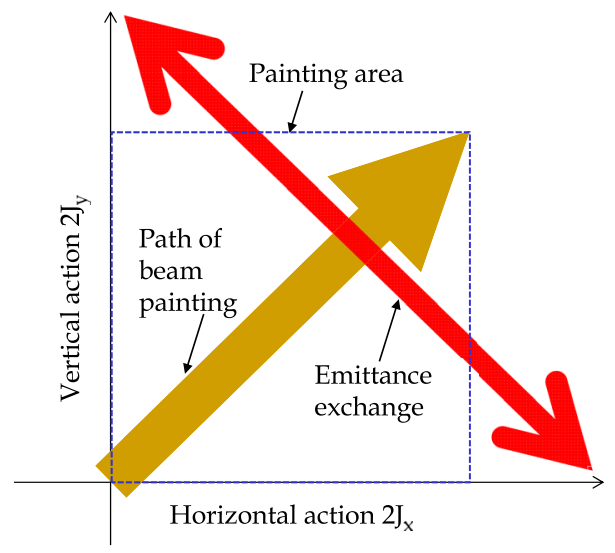

FIG. 6. Schematic illustration of the geometrical relation between correlated painting and emittance exchange in the $\left(J_{x}, J_{y}\right)$ space.

be seen that the space charge causes a significant diffusion of beam particles away from the path of beam painting, and it finally leads to a critical increase of the vertical action $J_{y}$ going over the painting area. This large diffusion of beam particles is mainly caused by space charge induced emittance exchange $\left(J_{x}-J_{y}\right.$ exchange of beam particles). As illustrated in Fig. 6, in correlated painting, the injection beam is painted along the line $J_{x}-J_{y}=0$, while emittance exchange occurs in the orthogonal direction, i.e., along the line $J_{x}+J_{y}=$ const. This geometrical relationship has the consequence that emittance exchange leads to a large diffusion of beam particles in the $\left(J_{x}, J_{y}\right)$ space. Figure 7 displays the single-particle motion of one macroparticle during correlated painting. This clearly shows that emittance exchange, which occurs perpendicularly to the path of beam painting, causes the diffusion of beam particles and the increase of the peak $J_{y}$ of beam particles found in the right panels of Fig. 5. This is the mechanism of the critical vertical emittance growth observed in Fig. 4 for correlated painting with $\epsilon_{t p}=200 \pi \mathrm{mm}$ mrad.

Figure 8 shows the case of anticorrelated painting with $\epsilon_{t p}=200 \pi \mathrm{mm} \mathrm{mrad}$. As shown clearly in the left panels
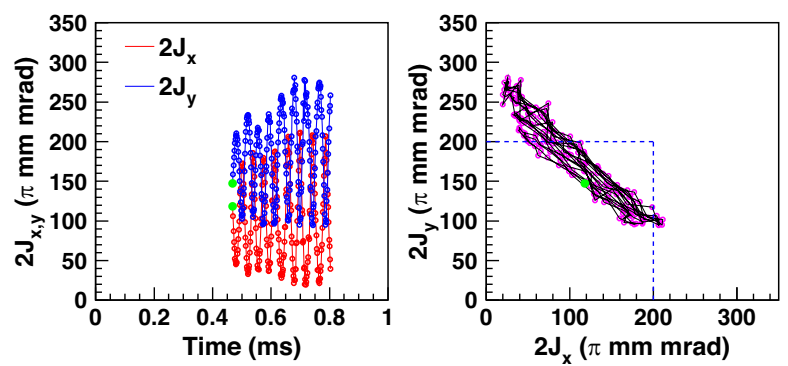

FIG. 7. Numerical simulation result; single-particle motion of one macroparticle during correlated painting with $\epsilon_{t p}=$ $200 \pi \mathrm{mm}$ mrad; $J_{x}$ and $J_{y}$ as a function of time (left), and their trajectory in the $\left(J_{x}, J_{y}\right)$ space (right). 

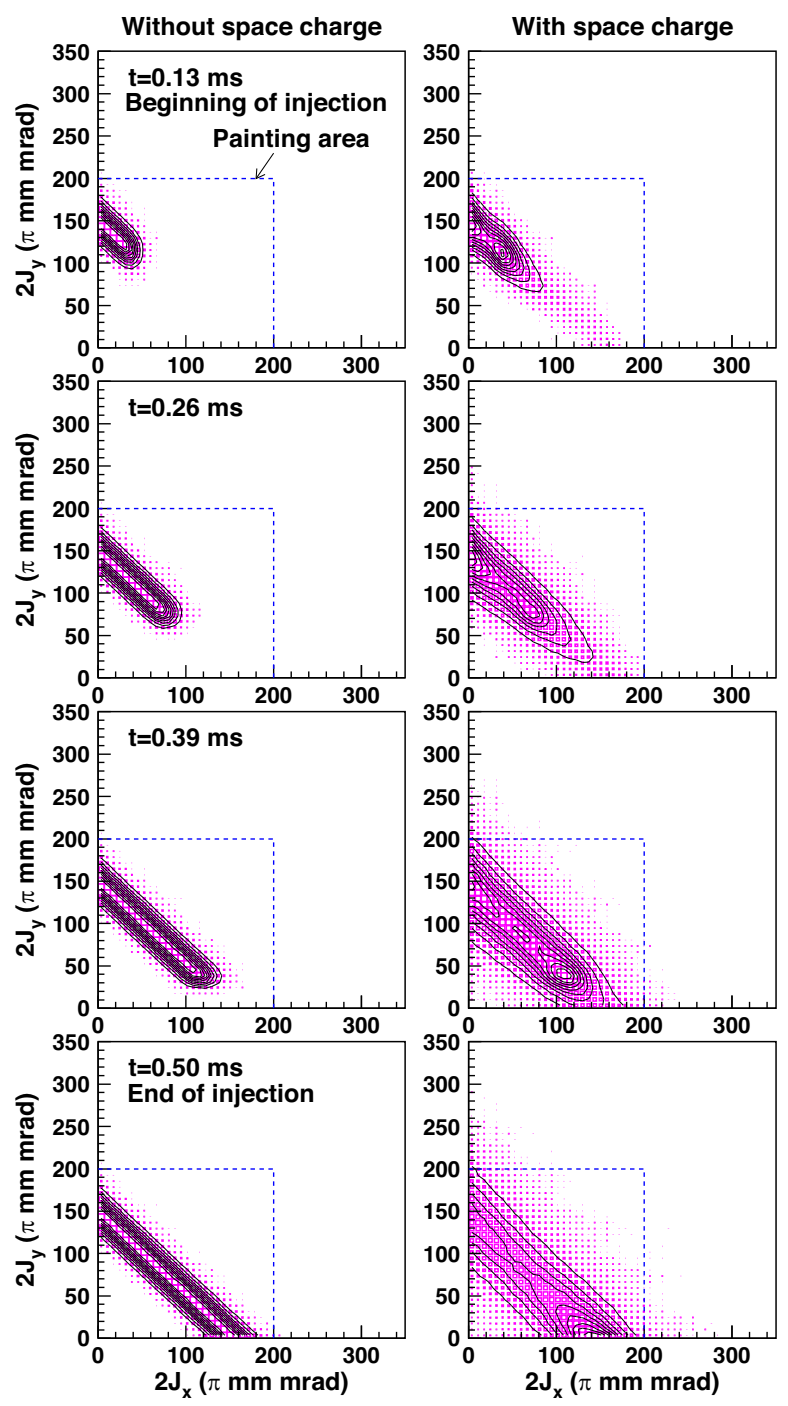

FIG. 8. Numerical simulation results; scatter plots of the singleparticle actions $\left(\begin{array}{llll}J_{y} & v s & J_{x}\end{array}\right)$ from the beginning to the end of injection, calculated for anticorrelated painting with $\epsilon_{t p}=$ $200 \pi \mathrm{mm}$ mrad without (left) and with (right) space charge.

obtained with no space charge, in anticorrelated painting, the injection beam is painted along the line $J_{x}+J_{y}=$ const, i.e., from the middle to the outside on the horizontal plane, while it is done from the outside to the middle on the vertical plane. This situation also changes significantly when the space charge is turned on, as shown in the right panels; it can be seen that the space charge induces emittance exchange along the same line $J_{x}+J_{y}=$ const. As illustrated in Fig. 9, the direction of anticorrelated painting is the same as that of emittance exchange. This geometrical relationship prevents emittance exchange from causing a large diffusion of beam particles in the $\left(J_{x}, J_{y}\right)$ space (a critical increase of the peak action of beam particles). In the right panels of Fig. 8, one can confirm that most of the beam particles stay in the painting area though emittance exchange occurs, because the beam

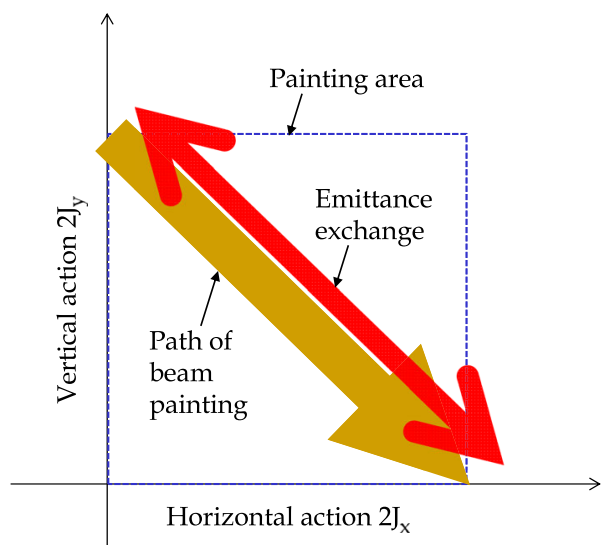

FIG. 9. Schematic illustration of the geometrical relation between anticorrelated painting and emittance exchange in the $\left(J_{x}, J_{y}\right)$ space.

painting and the emittance exchange take place in the same direction. Figure 10 shows the single-particle motion of one macroparticle during anticorrelated painting, which displays such a situation clearly.

Thus, emittance exchange has different effects on the formation of the beam distribution, depending on the geometrical relation in the $\left(J_{x}, J_{y}\right)$ space between beam painting and emittance exchange. The analysis above suggests that anticorrelated painting is more favorable for suppressing beam loss that arises from emittance exchange.

In Figs. 11 and 12, the numerical simulation results are compared with the experimental data from a 1-MW beam test. Simulated tune footprints are also given in Fig. 13 for reference. The numerical simulations well reproduced the characteristic beam profiles affected by the Montague resonance (Fig. 11), and also the beam losses including their amounts and time structures (Fig. 12). As shown in the left panel of Fig. 12, there was a significant beam loss-around $2.5 \%$-for correlated painting with $\epsilon_{t p}=200 \pi \mathrm{mm} \mathrm{mrad}$. As discussed above, the numerical simulations clearly demonstrated that the beam loss is due to the vertical emittance growth caused by the emittance exchange, finally reaching the collimator aperture of $324 \pi \mathrm{mm} \mathrm{mrad}$.
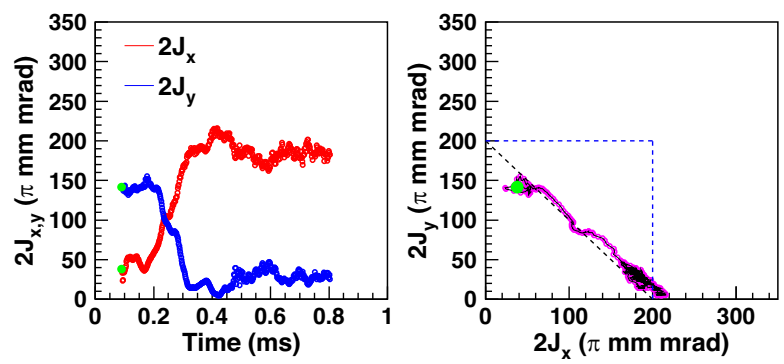

FIG. 10. Numerical simulation result; single-particle motion of one macroparticle during anticorrelated painting with $\epsilon_{t p}=$ $200 \pi \mathrm{mm} \mathrm{mrad} ; J_{x}$ and $J_{y}$ as a function of time (left), and their trajectory in the $\left(J_{x}, J_{y}\right)$ space (right). 

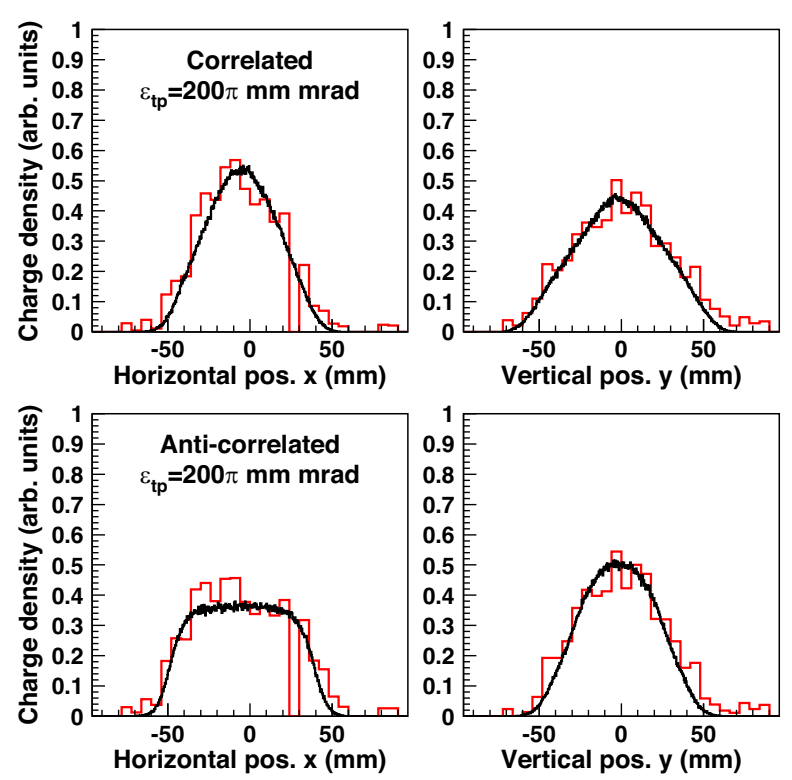

FIG. 11. (Red) Experimental results; transverse beam profiles right after injection, measured for correlated painting (top) and anticorrelated painting (bottom) with $\epsilon_{t p}=200 \pi \mathrm{mm} \mathrm{mrad}$. (Black) Corresponding numerical simulation results.

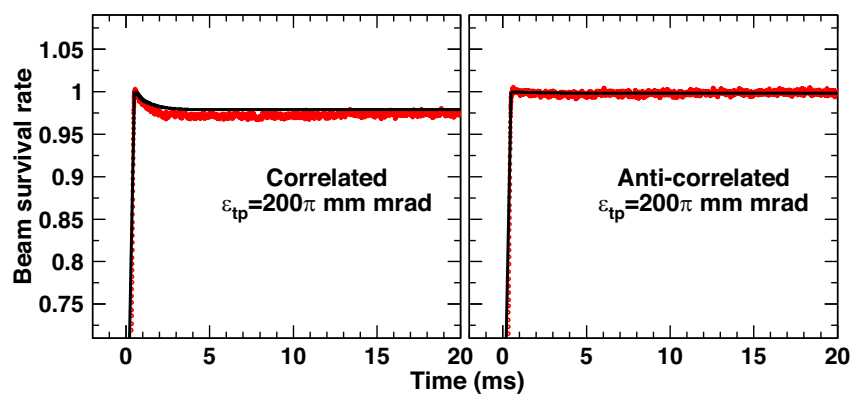

FIG. 12. (Red) Experimental results; beam survival rates from injection to extraction, measured for correlated painting (left) and anticorrelated painting (right) with $\epsilon_{t p}=200 \pi \mathrm{mm}$ mrad. (Black) Corresponding numerical simulation results.

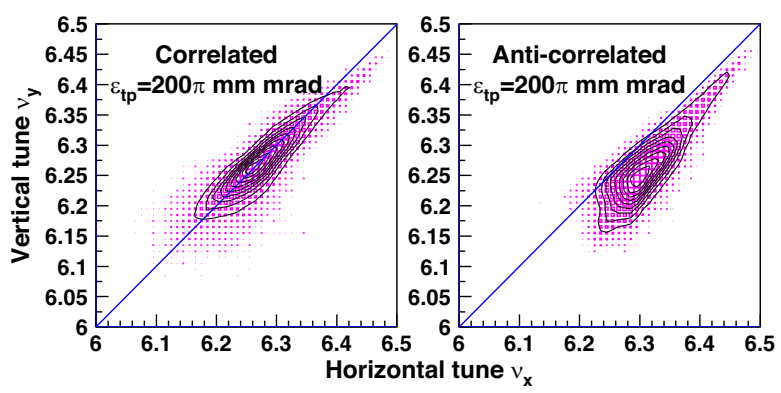

FIG. 13. Numerical simulation results; tune footprints at the end of injection, calculated for correlated painting (left) and anticorrelated painting (right) with $\epsilon_{t p}=200 \pi \mathrm{mm} \mathrm{mrad}$.
However, by introducing anticorrelated painting with the same painting emittance, we successfully reduced the beam loss to a few times $10^{-3}$, as predicted by the numerical simulations. The measurements and the numerical simulations both confirmed the advantage of anticorrelated painting, which has a higher tolerance for emittance exchange.

\section{EFFECTS OF EMITTANCE EXCHANGE DURING SMALL PAINTING}

Next, we discuss the case of small painting with $\epsilon_{t p}=50 \pi \mathrm{mmmrad}$, which is required for the beam delivered to the MR.

Figure 14 shows the normalized $99 \%$ emittances $1 \mathrm{~ms}$ after the end of injection, simulated as a function of the painted emittance $\epsilon_{t p}$ for both correlated and anticorrelated painting. This dependence reflects the balance between painting emittance and space-charge mitigation; the two are well-balanced at $\epsilon_{t p}=50 \pi \mathrm{mm} \mathrm{mrad}$, where the beam emittance is minimized. In addition, this figure shows that correlated painting, rather than anticorrelated painting, achieves smaller beam emittances on both the horizontal and vertical planes at $\epsilon_{t p}=50 \pi \mathrm{mm}$ mrad. This situation differs from the case of large painting with $\epsilon_{t p}=$ $200 \pi \mathrm{mm}$ mrad. This characteristic of small painting is also understandable by considering the effects of emittance exchange, as discussed below.

As already shown in Fig. 9, in anticorrelated painting, the direction of beam painting is the same as that of emittance exchange. This geometrical relationship suppresses emittance growth caused directly by emittance exchange itself, but it has the potential to cause a spatial region of high charge density. Figure 15 shows scatter plots of the single-particle actions $\left(J_{y} v s J_{x}\right)$ during anticorrelated
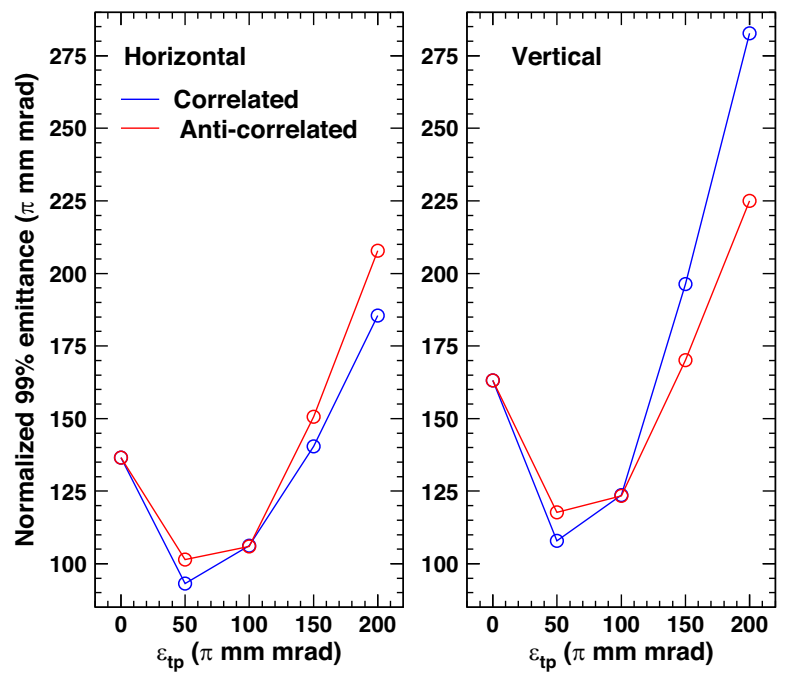

FIG. 14. Numerical simulation results; normalized 99\% emittances $1 \mathrm{~ms}$ after the end of injection, calculated as a function of the painting emittance for correlated and anticorrelated painting. 

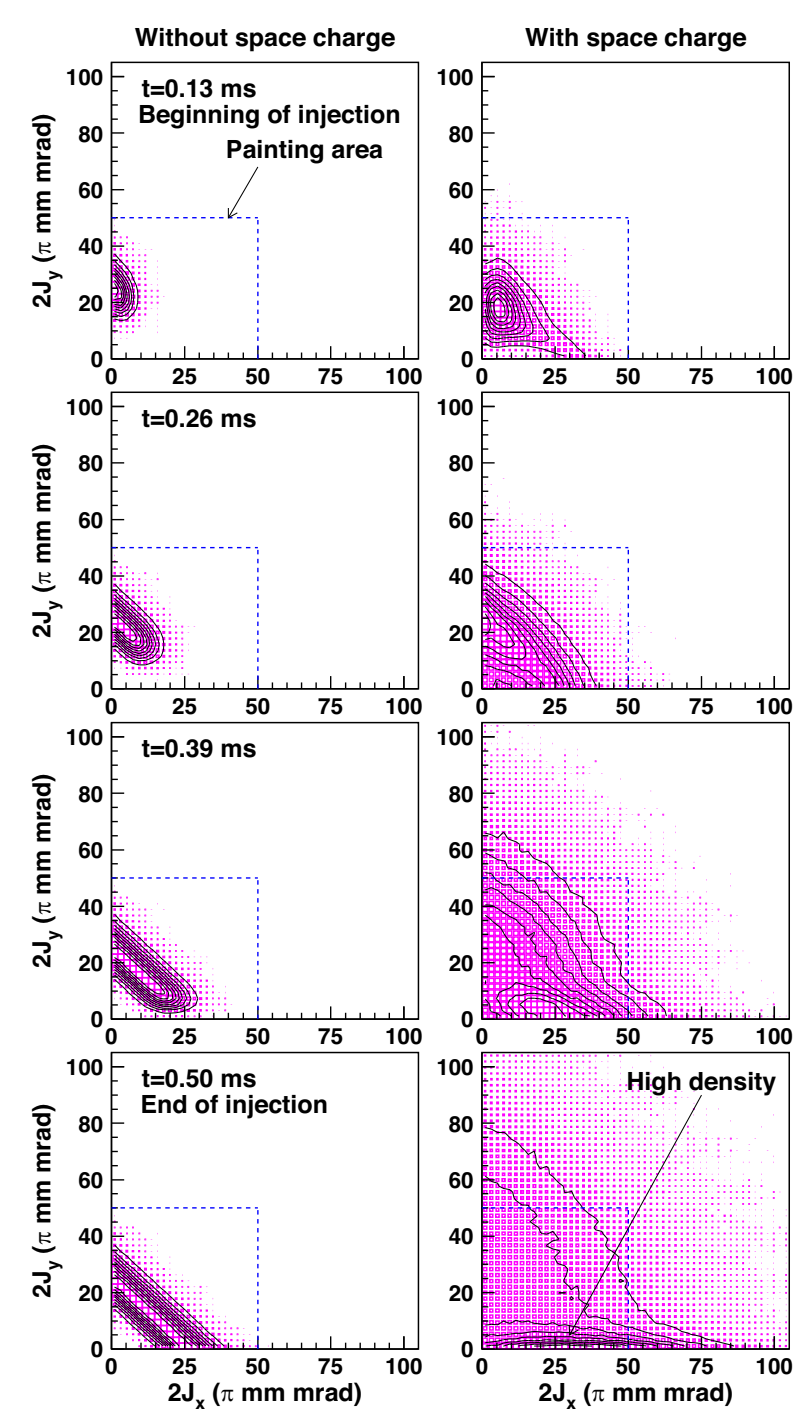

FIG. 15. Numerical simulation results; scatter plots of the single-particle actions ( $J_{y}$ vs $\left.J_{x}\right)$ from the beginning to the end of injection, calculated for anticorrelated painting with $\epsilon_{t p}=$ $50 \pi \mathrm{mm}$ mrad without (left) and with (right) space charge.

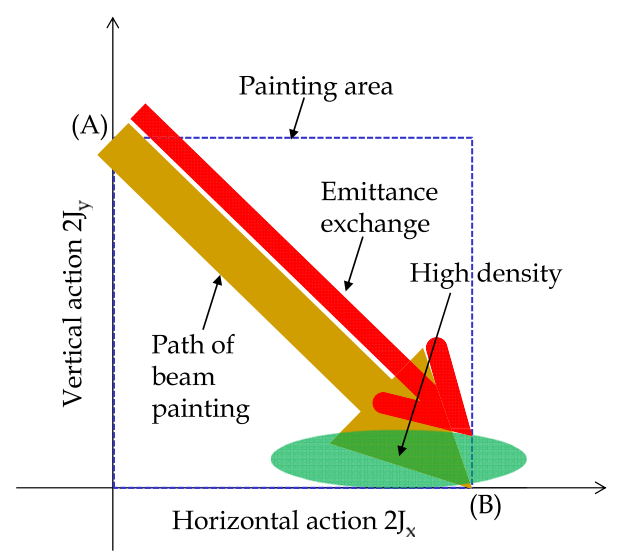

FIG. 16. Schematic illustration of the formation of a high-density island in anticorrelated painting with $\epsilon_{t p}=50 \pi \mathrm{mm}$ mrad.
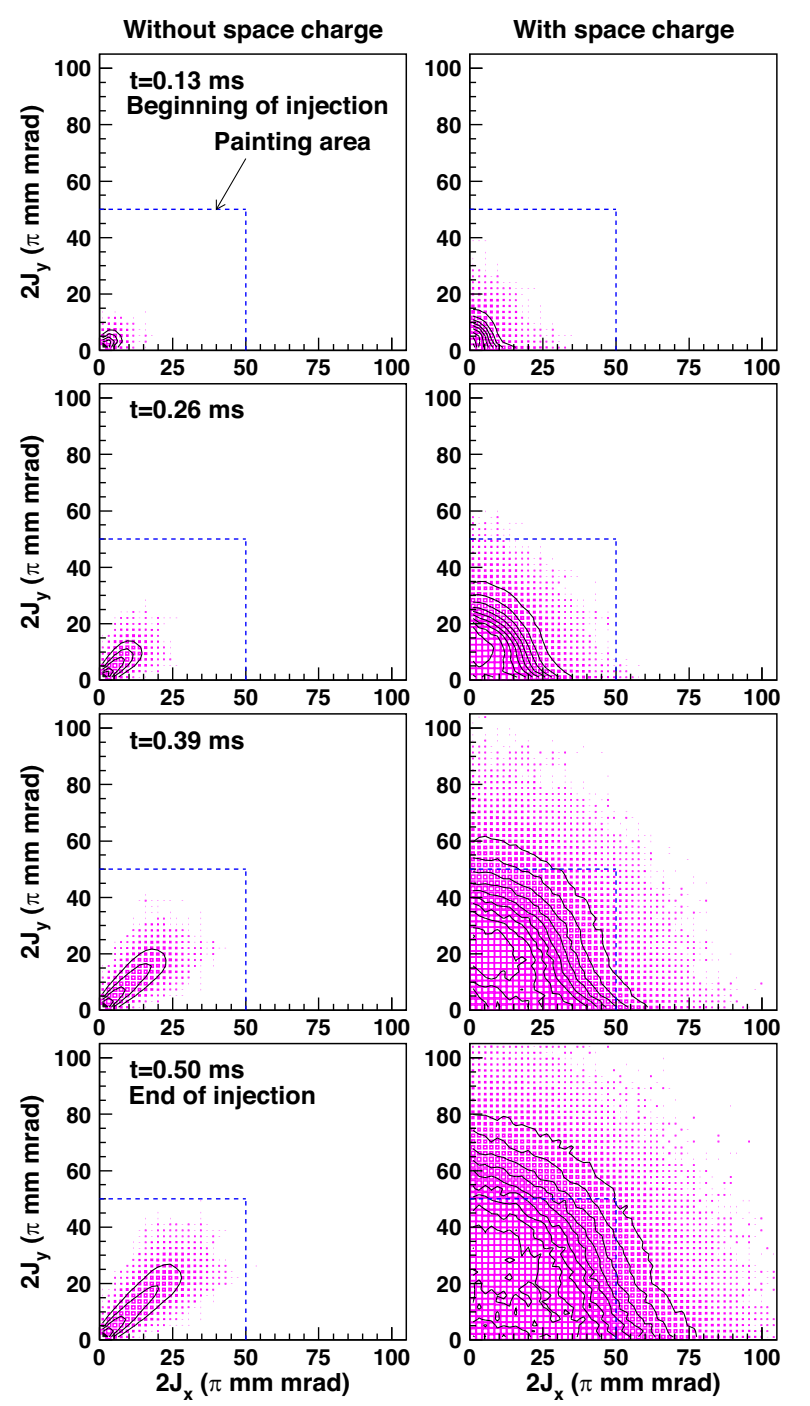

FIG. 17. Numerical simulation results; scatter plots of the single-particle actions $\left(J_{y}\right.$ vs $\left.J_{x}\right)$ from the beginning to the end of injection, calculated for correlated painting with $\epsilon_{t p}=$ $50 \pi \mathrm{mm}$ mrad without (left) and with (right) space charge.

painting with $\epsilon_{t p}=50 \pi \mathrm{mm}$ mrad. In this figure, one can find the formation of a high-density island at the late stage of injection. As illustrated in Fig. 16, in anticorrelated painting, the injection beam is painted from (A) to (B). Consequently, emittance exchange also starts from (A) and moves in the same direction so as to eliminate the emittance anisotropy between the two transverse planes early during injection. A specific link between the move of the beam distribution caused by the coherent emittance exchange and the beam painting produces this concentration of beam particles. This kind of high spatial charge concentration is not found in large painting, as shown in Fig. 8. Enlarging the painting emittance is equivalent to increasing the speed of beam painting. This has the effects of breaking the coordinated movements between emittance exchange and beam painting as well as improving space-charge mitigation. 

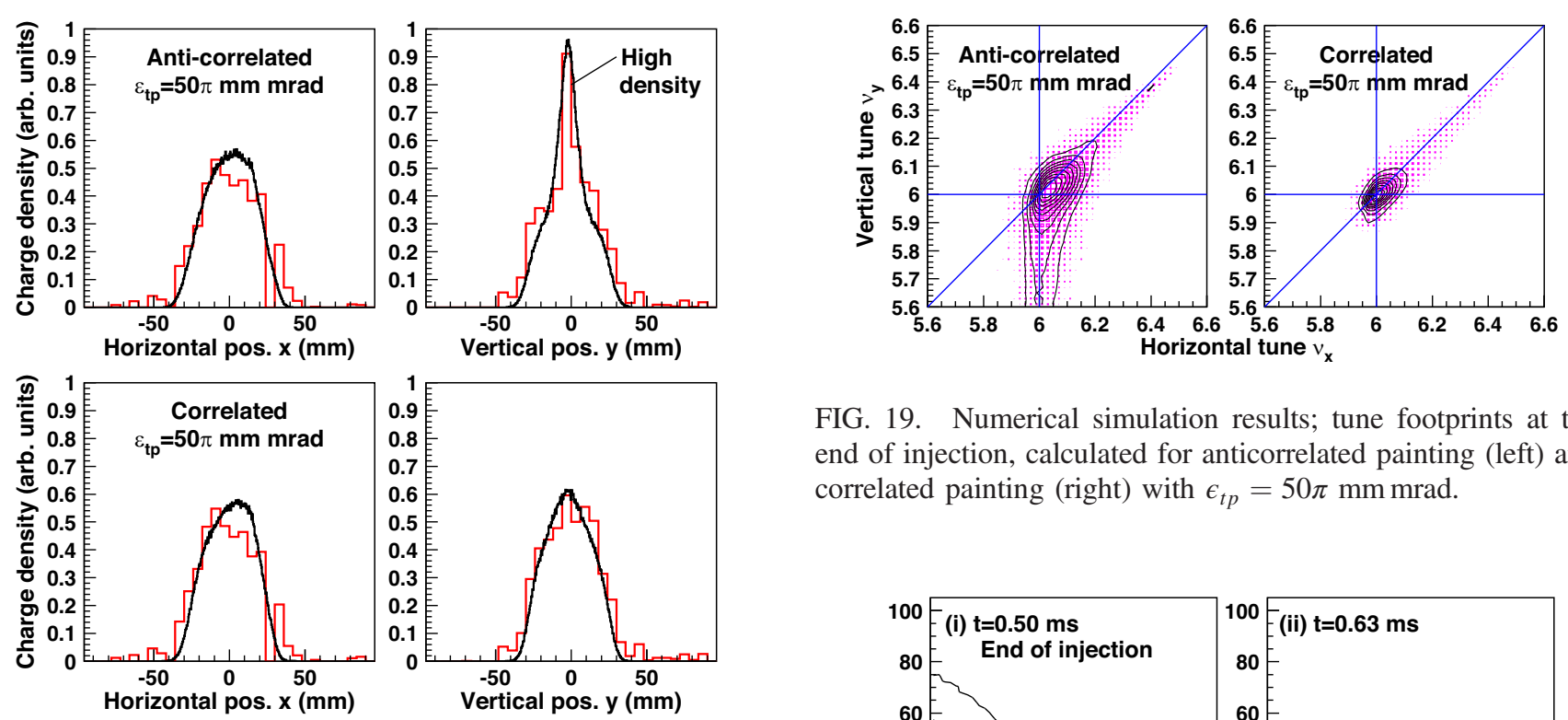

FIG. 19. Numerical simulation results; tune footprints at the end of injection, calculated for anticorrelated painting (left) and correlated painting (right) with $\epsilon_{t p}=50 \pi \mathrm{mm} \mathrm{mrad}$.

FIG. 18. (Red) Experimental results; transverse beam profiles at the end of injection, measured for anticorrelated painting (top) and correlated painting (bottom) with $\epsilon_{t p}=50 \pi \mathrm{mm} \mathrm{mrad}$. (Black) Corresponding numerical simulation results.

This is the reason why a relatively uniform distribution is maintained in large anticorrelated painting. That is, it can be said that the high spatial charge concentration triggered by emittance exchange is a characteristic phenomenon that is enhanced in anticorrelated painting with a small painting emittance.

Figure 17 shows the case of correlated painting with $\epsilon_{t p}=50 \pi \mathrm{mm} \mathrm{mrad}$. In correlated painting, emittance exchange occurs in the direction orthogonal to the path of beam painting. This geometrical relationship enhances emittance growth caused directly by emittance exchange itself, but, in exchange for that, it has the advantage of avoiding high spatial charge concentration. In Fig. 17, one can see that a more uniform distribution is maintained at all times during correlated painting.

This behavior of the beam particles during small painting was experimentally confirmed, as shown in Fig. 18; we found a high-density peak structure for anticorrelated painting with $\epsilon_{t p}=50 \pi \mathrm{mm} \mathrm{mrad}$, while we observed a more uniform beam distribution for correlated painting with $\epsilon_{t p}=50 \pi \mathrm{mm} \mathrm{mrad}$, as predicted by the numerical simulations.

The high-density island formed during anticorrelated painting with $\epsilon_{t p}=50 \pi \mathrm{mm}$ mrad causes a large spacecharge detuning, as shown in Fig. 19. These beam particles additionally suffer strong effects of several structure resonances-such as $\nu_{x}=6$ as well as the Montague resonance-which cause significant extra emittance growth. This is confirmed in Fig. 20, which shows the subsequent behavior of the beam particles that form the high-density island. In Fig. 20(i), the green dots correspond to particles

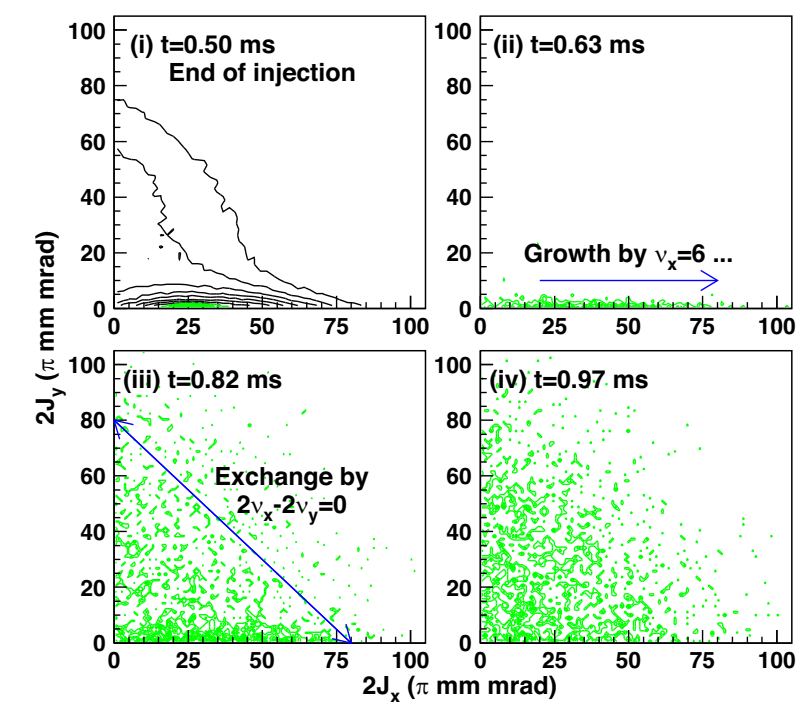

FIG. 20. Numerical simulation results; subsequent behavior of the beam particles that form the high-density island.

injected at the very end of the injection turns. They are injected right into the middle of the high-density island. These particles evolve in the order from (i) to (iv). As shown in the figure, the particles that form the high-density island diffuse quickly within just $\sim 300$ turns via a $J_{x^{-}}$ growth mainly by $\nu_{x}=6$ and the following $J_{x}-J_{y}$ exchange by $2 \nu_{x}-2 \nu_{y}=0$. This emittance blow-upcaused via the formation of the high-density island during anticorrelated painting - is more critical than that caused directly by emittance exchange itself during correlated painting. This is the main reason why anticorrelated painting leads to a larger emittance growth in small painting with $\epsilon_{t p}=50 \pi \mathrm{mm} \mathrm{mrad}$.

As shown in Fig. 21, there are no significant intensity losses for both correlated and anticorrelated painting with $\epsilon_{t p}=50 \pi \mathrm{mm} \mathrm{mrad}$, as the beam emittances are sufficiently small for both cases compared with the collimator aperture. But, from the perspective of minimizing beam loss in the following MR, the smaller-emittance beam, 


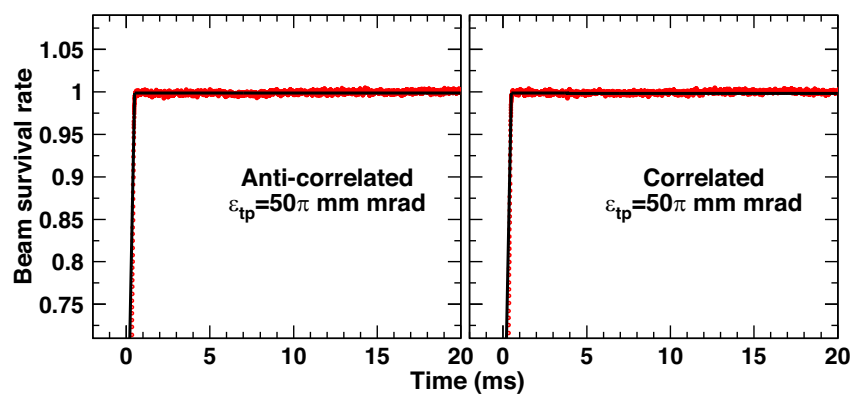

FIG. 21. (Red) Experimental results; beam survival rates from injection to extraction, measured for anticorrelated painting (left) and correlated painting (right) with $\epsilon_{t p}=50 \pi \mathrm{mm} \mathrm{mrad}$. (Black) Corresponding numerical simulation results.

which can be achieved by correlated painting, is of great value.

\section{SUMMARY OF RESULTS}

As described in Sec. I, the Montague resonance has both coherent and incoherent effects. Both beam distributions during correlated and anticorrelated painting intrinsically involve significant nonlinear space-charge coupling terms. Therefore, the incoherent effect occurs for both cases. On the other hand, the coherent effect is more significant for anticorrelated painting, because there is a large anisotropy between the horizontal and vertical emittances during this painting process. In contrast, for correlated painting, the coherent effect is less important, since correlated painting approximately maintains the isotropic condition $\epsilon_{x} \cong \epsilon_{y}$ throughout.

As discussed in the previous two sections, the resultant emittance exchange produces two major effects during injection painting. One (a) is emittance growth (increase of the peak action of beam particles) caused directly by the emittance exchange itself, which is more enhanced in correlated paining. Another (b) arises from the secondary effect of the emittance exchange, namely, emittance growth caused via high spatial charge concentration, which is more enhanced in anticorrelated painting. In large painting, such as $\epsilon_{t p}=200 \pi \mathrm{mm} \mathrm{mrad}$, the former effect (a) is more significant, so anticorrelated painting, which suppresses the effect (a), causes less beam loss. On the other hand, in small painting, such as $\epsilon_{t p}=50 \pi \mathrm{mm}$ mrad, the latter effect (b) is more critical, so correlated painting, which avoids the effect (b), causes less emittance growth.

On the basis of the present study results, we have successfully reoptimized the operating parametersincluding injection painting-for the MLF and the MR, and they are now applied for the routine user operations.

\section{FUTURE DEVELOPMENTS}

Finally, we discuss a possibility for a further improvement of injection painting based on the above study results.

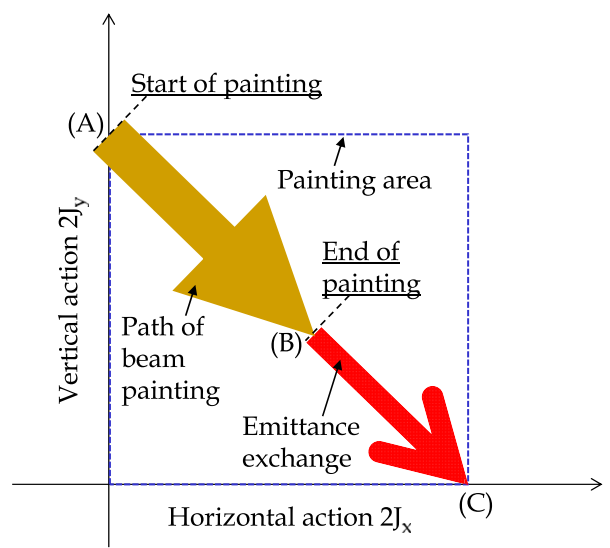

FIG. 22. Schematic illustration of anticorrelated painting modified to avoid high spatial charge concentration.
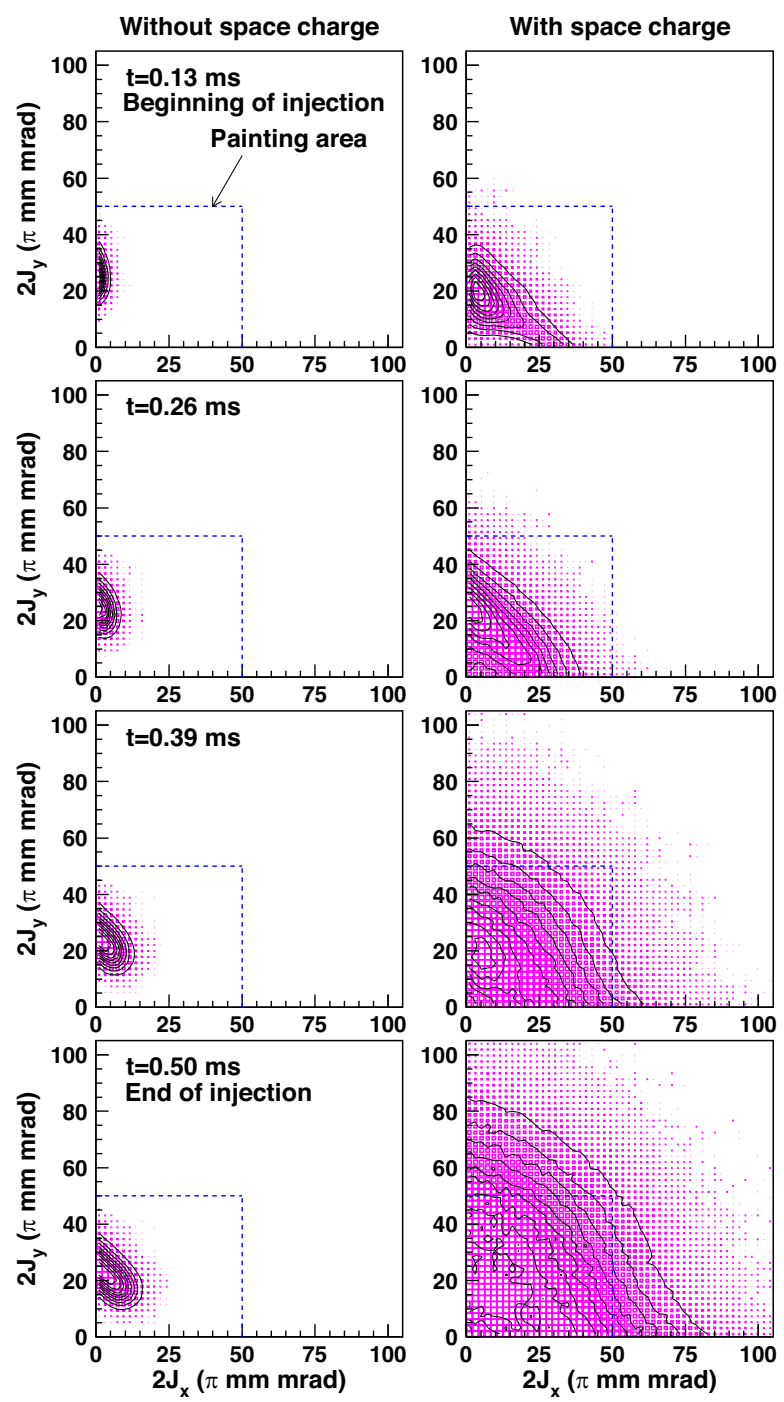

FIG. 23. Numerical simulation results; scatter plots of the single-particle actions $\left(J_{y}\right.$ vs $\left.J_{x}\right)$ from the beginning to the end of injection, calculated for modified anticorrelated painting with $\epsilon_{t p}=50 \pi \mathrm{mm} \mathrm{mrad}$ and $f_{\text {scl }}=0.4$ without (left) and with (right) space charge. 
Anticorrelated painting well suppresses emittance growth caused directly by emittance exchange itself, but it has the potential to cause high spatial charge concentration. One possible approach to removing this negative effect of anticorrelated painting is to adjust the range of beam painting in the $\left(J_{x}, J_{y}\right)$ space. One example is shown in Fig. 22, in which the injection beam is painted from (A) to (B), not from (A) to (C). In this modified manner, the phase-space offset of the injection beam relative to the closed ring orbit is varied as follows:

$$
\begin{aligned}
& x_{\text {paint }}=x_{\max } \sqrt{t / T \times f_{\text {scl }}}, \\
& x_{\text {paint }}^{\prime}=-x_{\max }^{\prime} \sqrt{t / T \times f_{\text {scl }}},
\end{aligned}
$$

for the horizontal plane, and

$$
\begin{aligned}
& y_{\text {paint }}=0, \\
& y_{\text {paint }}^{\prime}=-y_{\max }^{\prime} \sqrt{1-t / T \times f_{\text {scl }}},
\end{aligned}
$$

for the vertical plane, where $f_{\text {scl }}<1$ is a scaling factor. Comparison with Eqs. (4) and (6) shows that this modification is equivalent to reducing the speed of beam painting by a factor of $\sqrt{f_{\text {scl }}}$. In the empty space from (B) to (C), the beam is to be distributed automatically by emittance exchange.

Figure 23 shows scatter plots of the single-particle actions $\left(\begin{array}{lll}J_{y} & \text { vs } & J_{x}\end{array}\right)$ from the beginning to the end of injection, calculated for this modified anticorrelated painting with $\epsilon_{t p}=$ $50 \pi \mathrm{mm} \mathrm{mrad}$ and $f_{\text {scl }}=0.4$. Comparison with Fig. 15 shows that this scheme properly inhibits emittance exchange from causing high spatial charge concentration. As shown

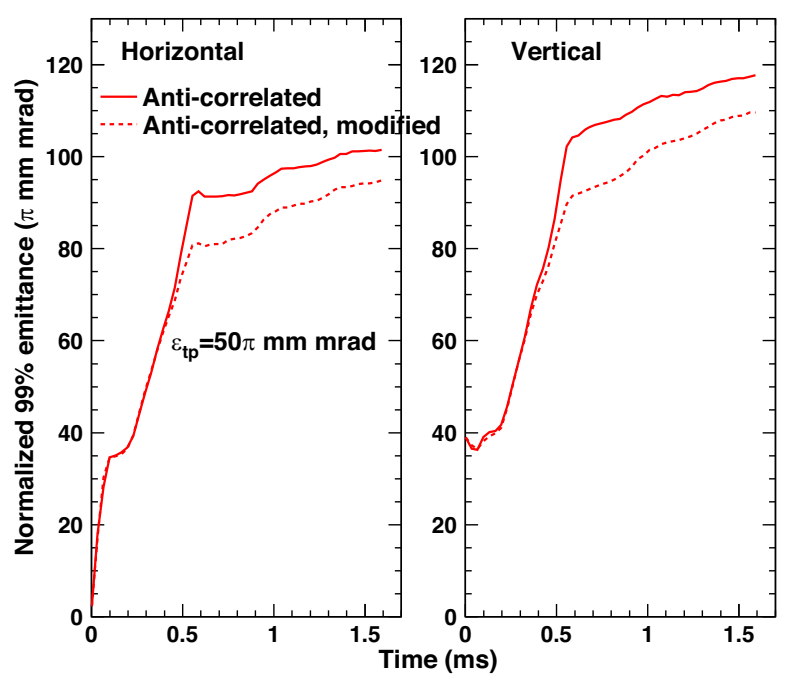

FIG. 24. Numerical simulation results; time dependences of the normalized $99 \%$ emittances for the first $1.5 \mathrm{~ms}$, calculated for both normal and modified anticorrelated painting with $\epsilon_{t p}=50 \pi \mathrm{mm} \mathrm{mrad}$. in Fig. 24, the numerical simulations confirmed that this scheme has the potential to realize further mitigation of emittance growth.

These numerical simulations suggest that injection painting has room for further improvement; modifying a time dependence of painting (e.g., linear or cubic root function), changing a balance between the horizontal and vertical painting emittances (i.e., unequal painting emittances), etc. Optimizing them is a subject for future investigations.

\section{SUMMARY}

We investigated the effects of space charge induced emittance exchange on injection painting in the 1-MWequivalent high-intensity environment of $8.33 \times 10^{13}$ protons per pulse. In this work, we found that the emittance exchange produces two major effects during injection painting: (a) Emittance growth (increase of the peak action of beam particles) caused by the direct effect of the emittance exchange itself, and (b) Emittance growth caused by the secondary effect of the emittance exchange, namely, via high spatial charge concentration.

These two effects are either enhanced or mitigated depending on the choice of correlated or anticorrelated painting and by the painting emittance. We also found that for a case involving emittance exchange, investigating the particle motions while considering the geometrical relationships between beam painting and emittance exchange in the $\left(J_{x}, J_{y}\right)$ space is to be the key to optimizing injection painting as well as to understanding the behavior of the beam. The choice of correlated or anticorrelated painting is a common issue in high-intensity proton rings like the RCS. The present work provides guidelines for solving this problem.

\section{ACKNOWLEDGMENTS}

The author acknowledges the core members of the RCS beam commissioning team, Dr. H. Harada, Dr. K. Okabe, Dr. P. K. Saha, Dr. Y. Shobuda, Dr. F. Tamura, and Dr. M. Yoshimoto, for their indispensable support during beam tests. The author is also grateful to Dr. N. Hayashi, Dr. K. Yamamoto, Dr. M. Kinsho, Dr. K. Hasegawa, Prof. T. Koseki and all the RCS group members for their continuous support and encouragement for the present work, and to Dr. S. Machida for his valuable advice and support for numerical simulations.

\section{APPENDIX: PERTURBATION HAMILTONIAN ARISING FROM THE SPACE CHARGE}

Here, the perturbation Hamiltonian arising from the space charge is derived and simplified based on Montague's approach [1] for reference. 
The total Hamiltonian $H$ can be divided into two parts as

$$
H\left(x, x^{\prime}, y, y^{\prime} ; s\right)=H_{0}+H_{1},
$$

where $\left(x, x^{\prime}=d x / d s\right)$ and $\left(y, y^{\prime}=d y / d s\right)$ are the horizontal and vertical phase-space coordinates, and $s$ is the longitudinal position along the closed ring orbit. In Eq. (A1), $H_{0}$ stands for the Hamiltonian of the unperturbed system in the absence of space charge; it can be written simply as

$H_{0}\left(x, x^{\prime}, y, y^{\prime} ; s\right)=\frac{1}{2}\left[x^{\prime 2}+k_{x}(s) x^{2}\right]+\frac{1}{2}\left[y^{\prime 2}+k_{y}(s) y^{2}\right]$,

where $k_{x}(s)$ and $k_{y}(s)$ represent the lattice focusing functions. As is well known, the general solution for the unperturbed motion is

$$
\begin{aligned}
& x=J_{x}^{1 / 2}\left[w_{1}(s) e^{i\left(\nu_{x} \theta+\varphi_{x}\right)}+\overline{w_{1}}(s) e^{-i\left(\nu_{x} \theta+\varphi_{x}\right)}\right], \\
& y=J_{y}^{1 / 2}\left[u_{1}(s) e^{i\left(\nu_{y} \theta+\varphi_{y}\right)}+\overline{u_{1}}(s) e^{-i\left(\nu_{y} \theta+\varphi_{y}\right)}\right],
\end{aligned}
$$

where $\left(\varphi_{x}, J_{x}\right)$ and $\left(\varphi_{y}, J_{y}\right)$ are conjugate pairs of the angle and action variables, $\left(\nu_{x}, \nu_{y}\right)$ are the betatron wave numbers, and $\theta=s / R$ is the orbiting angle, where $R$ is the ring radius. On the other hand, $H_{1}$ represents the perturbation part, including both linear and nonlinear space-charge terms. Assuming a uniform distribution with a line charge density $\lambda e$ in the longitudinal direction and Gaussian distributions in the horizontal and vertical directions as

$$
\begin{gathered}
f(x, y)=\frac{\lambda e}{2 \pi a b} e^{-\left(\frac{x^{2}}{2 a^{2}}+\frac{y^{2}}{2 b^{2}}\right)}, \\
4 \int_{0}^{\infty} \int_{0}^{\infty} f(x, y) d x d y=\lambda e,
\end{gathered}
$$

the Hamiltonian $H_{1}$ is expressed as

$$
\begin{aligned}
H_{1}\left(x, x^{\prime}, y, y^{\prime} ; s\right)= & \frac{\lambda r_{p}}{\beta^{2} \gamma^{3}}\left[-\frac{1}{a(a+b)} x^{2}-\frac{1}{b(a+b)} y^{2}\right. \\
& +\frac{2 a+b}{12 a^{3}(a+b)^{2}} x^{4}+\frac{a+2 b}{12 b^{3}(a+b)^{2}} y^{4} \\
& \left.+\frac{1}{2 a b(a+b)^{2}} x^{2} y^{2} \cdots\right] \\
\equiv & \sum_{k_{1}, k_{2}} V_{k_{1} k_{2}}(s) x^{k_{1}} y^{k_{2}},
\end{aligned}
$$

where $r_{p}$ is the classical proton radius, and $\beta$ and $\gamma$ are the relativistic Lorentz factors.
Using Eqs. (A3) and (A4), the Hamiltonian can be transformed into the angle-action coordinates; i.e., Eqs. (A3) and (A4) can be considered as a canonical transformation from $\left(x, x^{\prime}, y, y^{\prime}\right)$ to $\left(\varphi_{x}, J_{x}, \varphi_{y}, J_{y}\right)$. Substituting Eq. (A3) into the power series in $x$ in Eq. (A7), we obtain

$$
\begin{aligned}
x^{k_{1}}= & J_{x}^{k_{1} / 2}\left[w_{1}(s) e^{i\left(\nu_{x} \theta+\varphi_{x}\right)}+\overline{w_{1}}(s) e^{-i\left(\nu_{x} \theta+\varphi_{x}\right)}\right]^{k 1} \\
= & J_{x}^{k_{1} / 2} \sum_{r_{1}=0}^{k_{1}}\left(\begin{array}{c}
k_{1} \\
r_{1}
\end{array}\right) w_{1}^{\left(k_{1}-r_{1}\right)} e^{i\left(k_{1}-r_{1}\right)\left(\nu_{x} \theta+\varphi_{x}\right)} \\
& \times \bar{w}_{1}^{r_{1}} e^{-i r_{1}\left(\nu_{x} \theta+\varphi_{x}\right)},
\end{aligned}
$$

where $\left(\begin{array}{l}k_{1} \\ r_{1}\end{array}\right)$ is the binomial coefficient defined as $k_{1}\left(k_{1}-1\right) \cdots\left(k_{1}-r_{1}+1\right) / r_{1}$ !. Applying $k_{1}=l_{1}+m_{1}$ and $r_{1}=m_{1}$ as new notations, Eq. (A8) becomes

$x^{k_{1}}=J_{x}^{k_{1} / 2} \sum_{m_{1}=0}^{k_{1}=l_{1}+m_{1}}\left(\begin{array}{c}l_{1}+m_{1} \\ m_{1}\end{array}\right) w_{1}^{l_{1}}{\overline{w_{1}}}^{m_{1}} e^{i\left(l_{1}-m_{1}\right)\left(\nu_{x} \theta+\varphi_{x}\right)}$.

Similarly, we obtain

$y^{k_{2}}=J_{y}^{k_{2} / 2} \sum_{m_{2}=0}^{k_{2}=l_{2}+m_{2}}\left(\begin{array}{c}l_{2}+m_{2} \\ m_{2}\end{array}\right) u_{1}^{l_{2}}{\overline{u_{1}}}^{m_{2}} e^{i\left(l_{2}-m_{2}\right)\left(\nu_{y} \theta+\varphi_{y}\right)}$.

Substituting Eqs. (A9) and (A10) into Eq. (A7), the Hamiltonian $H_{1}$ becomes

$$
\begin{aligned}
H_{1}\left(\varphi_{x}, J_{x}, \varphi_{y}, J_{y} ; s\right)= & \sum_{k_{1}, k_{2}} \sum_{m_{1}}^{l_{1}+m_{1}} \sum_{m_{2}}^{l_{2}+m_{2}} V_{l_{1} m_{1} l_{2} m_{2}} J_{x}^{k_{1} / 2} J_{y}^{k_{2} / 2} \\
& \times e^{i\left[\left(l_{1}-m_{1}\right)\left(\nu_{x} \theta+\varphi_{x}\right)+\left(l_{2}-m_{2}\right)\left(\nu_{y} \theta+\varphi_{y}\right)\right],}
\end{aligned}
$$

where

$$
\begin{aligned}
V_{l_{1} m_{1} l_{2} m_{2}}= & \left(\begin{array}{c}
l_{1}+m_{1} \\
m_{1}
\end{array}\right)\left(\begin{array}{c}
l_{2}+m_{2} \\
m_{2}
\end{array}\right) \\
& \times w_{1}^{l_{1}} \bar{w}_{1} m_{1} u_{1}^{l_{2}} \bar{u}_{1}^{m_{2}} V_{k_{1} k_{2}} .
\end{aligned}
$$

Here and in Eqs. (A3) and (A4), the quantities $2\left|w_{1}\right|^{2}$ and $2\left|u_{1}\right|^{2}$ represent the betatron amplitude functions, which are given in the smooth approximation as

$$
\begin{gathered}
w_{1}=\overline{w_{1}}=\sqrt{\frac{R}{2 \nu_{x}}}, \\
u_{1}=\overline{u_{1}}=\sqrt{\frac{R}{2 \nu_{y}}} .
\end{gathered}
$$


Substituting Eqs. (A13) and (A14) into Eq. (A12), we get

$$
\begin{aligned}
V_{l_{1} m_{1} l_{2} m_{2}}= & \left(\begin{array}{c}
l_{1}+m_{1} \\
m_{1}
\end{array}\right)\left(\begin{array}{c}
l_{2}+m_{2} \\
m_{2}
\end{array}\right) \\
& \times\left(\frac{R}{2 \nu_{x}}\right)^{\frac{l_{1}+m_{1}}{2}}\left(\frac{R}{2 \nu_{y}}\right)^{\frac{l_{2}+m_{2}}{2}} V_{k_{1} k_{2}} .
\end{aligned}
$$

The perturbation Hamiltonian in Eq. (A11) can be classified into: (I) zero-frequency terms, with $l_{1}-m_{1}=$ 0 and $l_{2}-m_{2}=0$; (II) low-frequency terms, with $\nu_{x}-$ $\nu_{y} \approx 0$ and $l_{1}-m_{1}+l_{2}-m_{2}=0$; and (III) high-frequency terms. The motions of the beam particles are well-approximated by the terms (I) and (II), so we here neglect the highfrequency terms (III). Applying this approximation and using $V_{0220}=V_{2002}$, the Hamiltonian is finally simplified as follows:

$$
\begin{aligned}
H_{1}\left(\varphi_{x}, J_{x}, \varphi_{y}, J_{y} ; s\right)= & V_{1100} J_{x}+V_{0011} J_{y}+V_{2200} J_{x}^{2} \\
& +V_{0022} J_{y}^{2}+V_{1111} J_{x} J_{y} \\
& +2 V_{2002} J_{x} J_{y} \cos \left[2\left(\nu_{x}-\nu_{y}\right) \theta\right. \\
& \left.+2\left(\varphi_{x}-\varphi_{y}\right)\right],
\end{aligned}
$$

where

$$
\begin{aligned}
& V_{1100}=-\frac{\lambda r_{p}}{\beta^{2} \gamma^{3}} \frac{R}{\nu_{x}} \frac{1}{a(a+b)}, \\
& V_{0011}=-\frac{\lambda r_{p}}{\beta^{2} \gamma^{3}} \frac{R}{\nu_{y}} \frac{1}{b(a+b)}, \\
& V_{2200}=\frac{\lambda r_{p}}{\beta^{2} \gamma^{3}} \frac{R^{2}}{\nu_{x}^{2}} \frac{2 a+b}{8 a^{3}(a+b)^{2}}, \\
& V_{0022}=\frac{\lambda r_{p}}{\beta^{2} \gamma^{3}} \frac{R^{2}}{\nu_{y}^{2}} \frac{2 b+a}{8 b^{3}(a+b)^{2}}, \\
& V_{1111}=\frac{\lambda r_{p}}{\beta^{2} \gamma^{3}} \frac{R^{2}}{\nu_{x} \nu_{y}} \frac{1}{2 a b(a+b)^{2}}, \\
& V_{2002}=\frac{\lambda r_{p}}{\beta^{2} \gamma^{3}} \frac{R^{2}}{4 \nu_{x} \nu_{y}} \frac{1}{2 a b(a+b)^{2}} .
\end{aligned}
$$

[1] B. W. Montague, Fourth-order coupling resonance excited by space-charge forces in a synchrotron, CERN Report No. 68-38, 1968.
[2] I. Hofmann, Stability of anisotropic beams with space charge, Phys. Rev. E 57, 4713 (1998).

[3] I. Hofmann, G. Franchetti, O. Boine-Frankenheim, J. Qiang, and R. D. Ryne, Space-charge resonances in two and three dimensional anisotropic beams, Phys. Rev. Accel. Beams 6, 024202 (2003).

[4] I. Hofmann and O. Boine-Frankenheim, Resonant Emittance Transfer Driven by Space Charge, Phys. Rev. Lett. 87, 034802 (2001).

[5] L. M. Kapchinsky and V. V. Vladimirsky, in Proceedings of the International Conference on High Energy Accelerators (CERN, Geneva, 1959), p. 278.

[6] E. Metral, C. Carli, M. Giovannozzi, M. Martini, R. Steerenberg, G. Franchetti, I. Hofmann, J Qiang, and R. D. Ryne, Intensity dependent emittance transfer studies at the CERN Proton Synchrotron, in Proceedings of the 9th European Particle Accelerator Conference, Lucerne, 2004 (EPS-AG, Lucerne, 2004), p. 1984, [http://accelconf.web .cern.ch/AccelConf/e04/].

[7] J. Qiang, R. D. Ryne, G. Franchetti, I. Hofmann, and E. Metral, Numerical simulation study of the Montague resonance at the CERN Proton Synchrotron, in Proceedings of the 3rd International Particle Accelerator Conference, New Orleans, LA, 2012 (IEEE, Piscataway, NJ, 2012), p. 2958.

[8] H. Hotchi, H. Harada, N. Hayashi, M. Kinsho, P. K. Saha, Y. Shobuda, F. Tamura, K. Yamamoto, M. Yamamoto, M. Yoshimoto, and Y. Irie, Beam loss reduction by injection painting in the 3-GeV rapid cycling synchrotron of the Japan Proton Accelerator Research Complex, Phys. Rev. Accel. Beams 15, 040402 (2012).

[9] J. A. Holmes, T. Gorlov, N. J. Evans, M. Plum, and S. Cousineau, Injection of a self-consistent beam with linear space charge force into a ring, Phys. Rev. Accel. Beams 21, 124403 (2018).

[10] High-intensity Proton Accelerator Project Team, Accelerator Technical Design Report for High-intensity Proton Accelerator Facility Project, J-PARC, JAERI Report No. JAERI-Tech 2003-044, 2003.

[11] H. Hotchi et al., Beam commissioning of the $3-\mathrm{GeV}$ rapid cycling synchrotron of the Japan Proton Accelerator Research Complex, Phys. Rev. Accel. Beams 12, 040402 (2009).

[12] H. Hotchi, H. Harada, N. Hayashi, S. Kato, M. Kinsho, K. Okabe, P. K. Saha, Y. Shobuda, F. Tamura, N. Tani, Y. Watanabe, K. Yamamoto, M. Yamamoto, and M. Yoshimoto, Achievement of a low-loss 1-MW beam operation in the $3-\mathrm{GeV}$ rapid cycling synchrotron of the Japan Proton Accelerator Research Complex, Phys. Rev. Accel. Beams 20, 060402 (2017).

[13] H. Hotchi, Y. Watanabe, H. Harada, K. Okabe, P. K. Saha, Y. Shobuda, F. Tamura, and M. Yoshimoto, Pulse-by-pulse switching of operational parameters in J-PARC $3-\mathrm{GeV}$ RCS, J. Phys. Conf. Ser. 1067, 052015 (2018).

[14] S. Machida and M. Ikegami, Simulation of space charge effects in a synchrotron, AIP Conf. Proc. 448, 73 (1998). 